\title{
Quantifying the subjective cost of self-control in humans
}

\author{
Candace M. Raio ${ }^{1} \&$ Paul W. Glimcher ${ }^{1}$ \\ ${ }^{1}$ Neuroscience Institute, New York University School of Medicine, \\ New York, NY, 10016 USA
}

*Please address all correspondence to:

Dr. Candace M. Raio

Neuroscience Institute

New York University School of Medicine

435 East $30^{\text {th }}$ Street

New York, NY 10016

Email: cmr385@nyu.edu 


\section{ABSTRACT}

Since Odysseus committed to resisting the Sirens, mechanisms to limit selfcontrol failure have been a central feature of human behavior. Psychologists have long argued that the use of self-control is an effortful process and, more recently, that its failure arises when the cognitive costs of self-control outweigh its perceived benefits. In a similar way, economists have argued that sophisticated choosers can adopt "pre-commitment strategies" that tie the hands of their future selves in order to reduce these costs. Yet, we still lack an empirical tool to quantify and demonstrate the cost of self-control. Here, we develop and validate a novel economic decision-making task to quantify the subjective cost of selfcontrol by determining the monetary cost a person is willing to incur in order to eliminate the need for self-control. We find that humans will pay to avoid having to exert self-control in a way that scales with increasing levels of temptation and that these costs are modulated both by motivational incentives and stress exposure. Our psychophysical approach allows us to index moment-to-moment self-control costs at the within-subject level, validating important theoretical work across multiple disciplines and opening new avenues of self-control research in healthy and clinical populations. 


\section{INTRODUCTION}

2 When Odysseus tied himself to the mast of his ship so he could hear the song of

3 the Sirens without approaching them, he deployed a pre-commitment mechanism

4 that prevented a self-control failure. When his men were unable to leave the land

5 of the lotus eaters, Homer urges us to see them as having failed in their self-

6 control. But what does it mean for self-control to fail? This has been a central

7 debate in human behavior for centuries. What has fueled this debate is not a

8 failure to understand what self-control feels like; the subjective experience of

9 resisting temptation is a universal one for humans. What has made self-control

10 so elusive is determining how to convincingly and quantitatively measure it, and

11 therefore to understand why it often fails. Whether we are trying to lose weight,

12 quit smoking, avoid drugs, exercise more, drink less, or simply focus on a

13 cognitively demanding task, the question remains: If one truly desires a particular

14 long-term outcome, why is it so difficult to choose in favor of that outcome all of

15 the time?

16 Emerging theoretical accounts from psychology and economics have attempted

17 to untangle this question using economic models of 'cost'. Exercising self-control,

18 these accounts propose, is cognitively costly. From this perspective, 'failures' of

19 self-control arise from a rational decision process that weighs the benefits of

20 exercising control against its attendant costs. That is, when the cognitive costs

21 exceed their perceived benefit, individuals should disengage from control

22 processes. These 'control costs' are thought to stem from the limited cognitive

23 resources available to support the demands of exercising control. As evidence of

24 these costs, economic theories point to the fact that choosers often adopt pre-

25 commitment strategies, presumably in an effort to reduce the need for self-

26 control (e.g., Strotz, 1956; Thaler \& Sherfin, 1981; Gul and Pesendorfer,

27 2001/2004). Yet, the notion of self-control as 'costly' remains controversial in the

28 absence of a robust psychophysical methodology for reliably demonstrating and

29 measuring these costs. 
1 Historically, theoretical accounts that have attempted to explain this puzzling

2 disconnect between what we say we want and what we actually do, have pointed

3 to the existence of self-control without providing a platform for its reliable

4 demonstration and quantification. The first, emerging from the psychological

5 literature, points to self-control as a top-down regulatory process that inhibits

6 impulsive action in the service of long-term goals or social norms. Informed by

7 findings from classic delay-of-gratification paradigms (e.g., the 'marshmallow

8 task'; Mischel \& Ebbesen, 1970; Mischel, Ebbesen, \& Zeiss, 1972; Mischel,

9 Shoda, and Rodriguez, 1989; but see also McGuire \& Kable, 2013) and theories

10 of ego depletion (Baumeister, Bratslavsky, Muraven, \& Tice, 1998; Muraven,

11 Tice, \& Baumeister, 1998; Baumeister \& Heatherton, 1996; Muraven \&

12 Baumeister, 2000; but also see Kurzban and colleagues, 2013), this account

13 proposes that self-control relies on cognitive resources that are depleted the

14 longer they are used. These theories suggest that the motivational or affective

15 state of a chooser influences the availability or functional integrity of these

16 resources. Fatigued or stressed choosers, for example, are often presumed to

17 have more limited cognitive resources for self-control upon which to draw

18 (Muraven, Tice, \& Baumeister, 1998; Arnsten, 2009; Hockey, 1983; Holding,

19 1983). This body of work has dominated psychological conceptions of self-control

20 as a form of 'willpower' with impulsive or suboptimal choice emerging from a

21 failure or depletion of control resources. While this work aptly captures the

22 subjectively difficult nature of exercising self-control, it has not provided a reliable

23 method to quantify how much control is needed to successfully resist temptation.

24 A second account from the neoclassical economic literature examines a host of

25 related choice problems including the failure to save money, over-consumption

26 and procrastination (see Ariely \& Wertenbroch, 2002; Bryan, Karlan \& Nelson,

272010 for behavioral examples). These economic models view self-control

28 'failures' as simple preference reversals (i.e., Strotz, 1956; Mischel \& Ebbesen, 
$1 \quad$ 1970; Ainslie, 1975; Schelling, 1984; Fishburn \& Rubinstein, 1982; Bryan, Karlan

2 \& Nelson, 2010), and have generally eschewed the psychological notion of self-

3 control as a hidden, and perhaps unnecessary, element. When individuals

4 choose in ways that conflict with explicitly stated goals, these choosers are seen

5 as revealing their true preferences through their observed choices. If this is the

6 case however, why do individuals often choose in ways that conflict with

7 previously stated goals, even choosing in ways that appear inconsistent or

8 irrational (Strotz, 1955; Thaler \& Sherfin, 1981; Tversky \& Thaler, 1990)? Some

9 behavioral economic work has accounted for this paradox with dual-self models

10 that propose that choosers possess (at least) two sets of preferences that are in

11 active competition (Thaler \& Sherfin, 1981; Fudenberg \& Levine; 2006), and

12 temporal discounting models that include dynamic inconsistencies to drive

13 changing preferences (Mazur, 1987; Ainslie, 1975; Laibson, 1997; Frederick et

14 al., 2002; but see Kable \& Glimcher, 2007/2010 for an alternate account).

15 While these models have provided important ways to quantify decision variables

16 related to self-control, they do not fully explain why individuals are inconsistent in

17 their actual choices. One widely influential resolution of this cross-disciplinary

18 puzzle is to hypothesize that the experience of resisting immediate temptation is

19 effortful and aversive - that is disutile. This inherent disutility implies that self-

20 control imposes a cost on choosers, an idea formalized most recently and

21 elegantly by Gul and Pesendorfer $(2001,2004)$ who proposed an axiomatic

22 model of self-control. Gul and Pesendorfer proposed that the presence of

23 temptation in an individual's 'menu' of choices will impose a cognitive cost,

24 rendering decisions to reject tempting options more difficult. They hypothesized

25 that if choosers know this, they should prefer choice menus that lack tempting

26 options and might even seek to minimize control costs (and maximize utility) by

27 preemptively eliminating tempting options from their choice menu, a

28 phenomenon referred to as 'pre-commitment' (Strotz, 1956; Thaler \& Sherfin,

29 1981; see Bryan, Karlan \& Nelson, 2010 for review). Examples of pre- 
1 commitment strategies that limit control costs might include a dieter who is willing

2 to walk an extra block to avoid a local bakery or a gambler who drives an extra

3 hour to avoid casinos. Gul and Pesendorfer thus argued that preferences for pre-

4 commitment reveal choosers' subjective cost of exercising control, pointing to a

5 novel decision variable through which these costs can be measured.

6 What has limited the impact of this set of hypotheses in an empirical sense is the

7 absence of quantitative data to support it. Is there direct quantitative empirical

8 evidence that self-control is costly, as so many have proposed? Are those costs

9 stationary over time? Are these costs influenced by motivational or affective state

10 as the psychological literature has proposed? Do these costs scale with varying

11 levels of temptation? Despite a number of real-world observations of pre-

12 commitment (see Bryan, Karlan \& Nelson, 2010 for review), we still lack an

13 empirical psychophysical tool for answering these questions.

14 Here we utilized a psychophysical approach to test the hypothesis that exercising

15 self-control is cognitively costly and that these costs can be measured using a

16 pre-commitment mechanism. While we acknowledge that there are undoubtedly

17 many components that feed into an overall subjective cost of self-control, our

18 goal here was to simply measure an aggregate of these costs. To do this, we

19 developed an economic decision-making task that measures how much

20 participants are willing to pay to adopt a pre-commitment device that removes

21 temptation from their choice environment. We refer to the maximum dollar value

22 participants will pay to remove temptation as their subjective 'cost of self-control'

23 and show that these costs respond rationally to incentives, scale with increasing

24 levels of temptation and predict rates of self-control failure. We further test the

25 hypothesis that these costs are modulated by affective state, finding that stress

26 exposure significantly increases the cost of self-control. Finally, we test the

27 hypothesis that self-control costs grow with the ongoing exertion of self-control

28 but find no empirical support for this hypothesis.-These data identify a

29 psychophysical approach for the measurement of self-control costs and may 
1 open new avenues of research into computational models of self-control to

2 inform psychological, economic, clinical and health policy research.

\section{RESULTS}

4 In our experiments, healthy, hungry dieters first provided health, taste and

5 temptation ratings for a series of food items, allowing us to identify high- and low-

6 tempting foods for each individual. Participants initially reported the most they

7 would be willing-to-pay (from a $\$ 10$ monetary endowment) to avoid having the

8 high-tempting food placed immediately in front of them for a 30-minute period

9 (Figure 1). With a probability of $2 \%$ this reported 'bid' was entered into a

10 standard economic auction procedure (see Methods; Becker, DeGroot \&

11 Marschak, 1964) that incentivizes participants to report their true subjective

12 value - in this case the value of eliminating exposure to temptation. If they won

13 this auction, the high-tempting food was replaced with a low-tempting food for 30

14 minutes. If they lost the auction, the high tempting food remained in the room

15 with them for 30 minutes. The price participants were willing to pay provided a

16 within-subject estimate of the cost of self-control; that is, it reflected the maximal

17 dollar value they were willing to pay to avoid exercising control.

19 The first bidding trial was made without the high tempting food in the room to

20 capture each participants' prospective estimate of how costly self-control exertion

21 would be (before exposure to temptation). If the initial trial was not realized (as

22 occurred on $98 \%$ of trials), the high-tempting food was brought into the room.

23 Participants were then prompted to report, periodically during 30-minutes of

24 exposure, how much they were willing-to-pay to replace the high-tempting food

25 with the low-tempting food for the next 30 minutes. As with the initial bid, these

26 subsequent bidding trials had a $2 \%$ chance of going to auction, which would

27 bring the experiment to a premature close. Bidding trials were collected every

28 few minutes (Methods) for the 30-minute exposure period. If the 30-minute

29 exposure period elapsed without any bid being realized, the subjects remained in 
1 the room with the high-tempting good for a final 30 minutes. This allowed us to

2 track how these self-control costs change over time as participants are

3 continuously exposed to temptation and whether self-control ever failed.

'Prospective control cost'

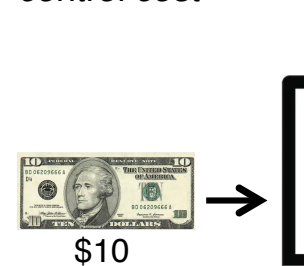

endowment

.
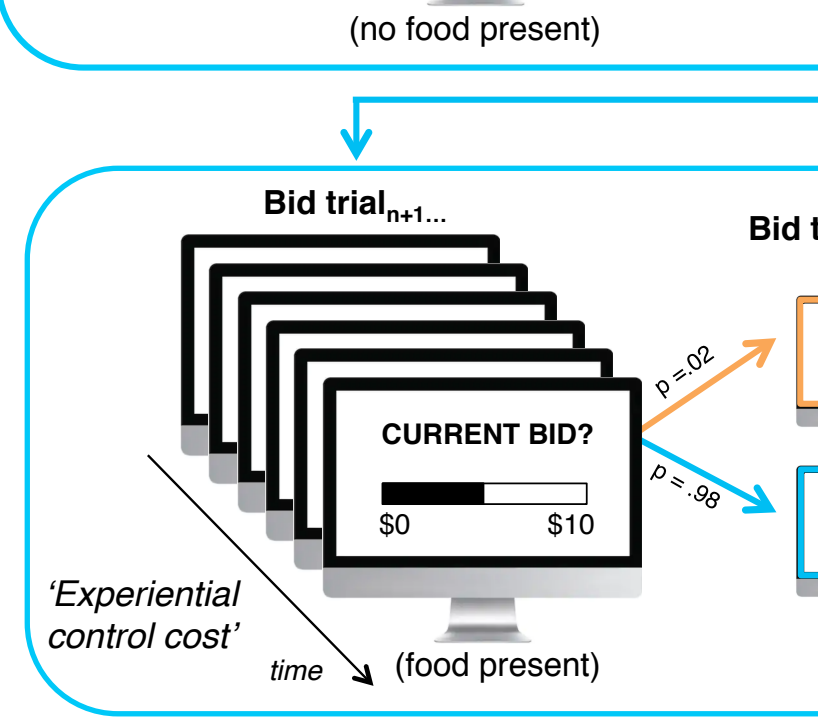

Bid trial realization

\section{Realization Phase}
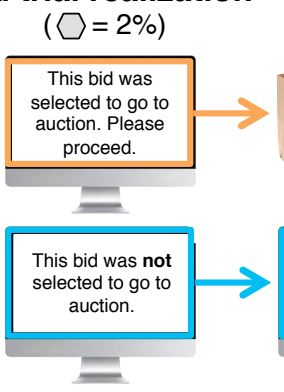

$=$

Bid trial realization
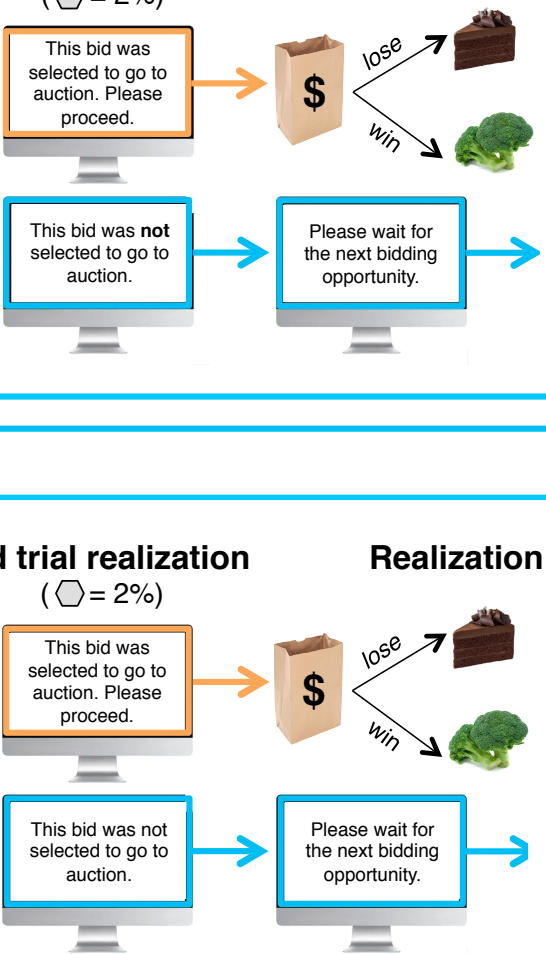

Bidding ends; 30 min with high- or low-tempting food

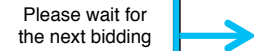

the next bidding

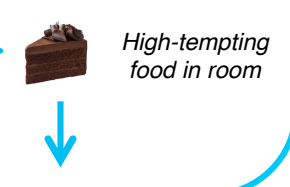

Realization Phase

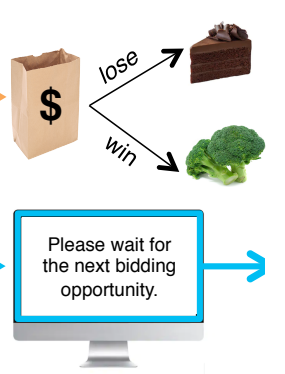

Bidding ends; High-tempting food remains in room for 30 min or is switched with low-tempting food

High-tempting food remains in room

Figure 1. Illustration of the self-control decision task. Participants reported their willingness-to-pay to avoid a tempting food reward both before the food was present (top panel) and, periodically over a 30-minute period, with added direct exposure to the food (bottom panel).

4 The only observable behavior of interest during the final 30-minute interval of the

5 experiment was whether or not the participant consumed the food. This

6 realization phase of the experiment ensured that it was incentive-compatible,

7 meaning participants' choices allowed them to avoid real temptations and the

8 negative outcomes associated with those temptations. The $2 \%$ chance of each 
1 bid being realized ensured participants knew that what they bid on the current

2 trial was important, since it could determine whether they were required to spend

3 the next 30 minutes alone in the experiment room with a tempting food reward,

4 the consumption of which did not align with their stated goals.

5 Study 1: Self-control imposes costs as revealed by willingness-to-pay for

6 pre-commitment. Our primary question of interest was whether the presence of

7 a tempting good participants want to avoid consuming does in fact impose a cost

8 on choosers. If so, participants should be willing to pay to remove temptation and

9 eliminate the associated control cost. In accord with predictions from these

10 economic models, we found that participants $(n=32)$ were willing to pay a

11 maximum of, on average, $15 \%$ of their $\$ 10$ endowment (or $\$ 1.57 \pm 1.78$ SD) to

12 adopt a pre-commitment device that avoided temptation. This provides a direct

13 scalar measurement of their subjective cost of resisting temptation (Figure 2A).

14 Participants were not only willing to pay to prospectively avoid temptation (mean

15 of first bid trial, pre-exposure $=\$ 1.47 \pm 1.59 \mathrm{SD})$, but they continued to pay

16 throughout the task, providing a continuous measurement of the underlying costs

17 of resisting temptation with continuing exposure to the food (mean of subsequent

18 bid trials, post-exposure $=\$ 1.58 \pm 1.82 \mathrm{SD}$ ).

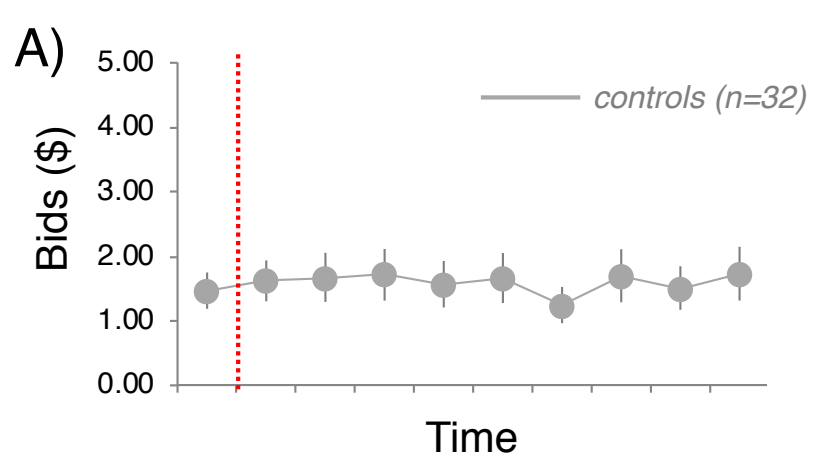

denotes onset of temptation exposure

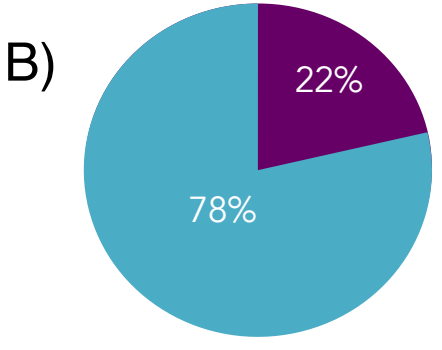

Self-control 'failures'

Figure 2. Study 1. (A) Bids over time for control group; (B) Proportion of subjects in Study 1 that consumed tempting food during the study. Error bars denote SEM. 
1 Our initial experiment thus demonstrates that self-control imposes a subjective

2 cost on choosers that can be measured monetarily. However, the process of

3 deploying self-control in the presence of temptation has been proposed to

4 change over time. Specifically, continued exposure to a tempting good is often

5 thought to increase the difficulty of exerting self-control. We next examined the

6 dynamics of how bids changed both before and after exposure to temptation

7 (Figure 2A). We found that average pre- versus post-exposure bids did not

8 significantly differ (paired samples t-test: $\mathrm{t}_{(31)}=-0.533, P=0.598, d=0.099$, two-

9 tailed), suggesting that our dieters appear to be accurate in their prospective cost

10 estimates. A repeated-measures ANOVA assessing post-exposure bids as a

11 function of time indicated that on average there were no significant linear trends

12 in these costs despite ongoing exposure to temptation $\left(F_{(4,122)}=0.722, P=0.576\right.$;

13 Greenhouse-Geisser correction factor, $\left.\varepsilon=0.30 ; \eta_{p}^{2}=0.023\right)$. Thus, we found no

14 evidence that, on average, ongoing exposure to temptation increased control

15 costs over the interval used in our task. We note, however, that individual

16 variability exists in our data set, such that some participants' bids increased

17 systematically while for others they decreased. Finally, we note that $22 \%$ of our

18 subjects consumed the tempting food during the ensuing 30-minute exposure

19 period (Figure 2B).

20 Study 2: Motivational incentives modulate willingness-to-pay for pre-

21 commitment. Next, we sought to both replicate this finding, and further test how

22 motivational incentives to sustain goal-directed behavior might affect these costs.

23 In this second experiment we increased the cost of self-control failure and

24 examined whether participants were then willing to pay more for pre-commitment

25 to reduce self-control failures. To do this, we repeated our study in a second

26 cohort of dieters but instructed participants that they would lose a $\$ 15$ bonus at

27 the end of the study if they consumed the tempting food at any point. We

28 hypothesized that by increasing the cost of self-control failure, the value of a pre-

29 commitment strategy that restricts temptation should be also be higher. 
2 Thirty-four new dieters completed our self-control measurement task with the

3 addition of this second monetary incentive. Dieters again showed a reliable and

4 consistent willingness-to-pay to avoid temptation (mean bid $=\$ 2.85 \pm 2.70 \mathrm{SD}$ ).

5 Consistent with our hypothesis, the addition of the $\$ 15$ cost for eating the

6 tempting food led to a higher willingness-to-pay for pre-commitment (Figure 3A).

7 Combining the data across experiments 1 and 2, a repeated-measures ANOVA

8 with a within-subject factor of time (bids 1-10) and a between-subject factor of

9 group (no incentive, incentive) revealed a main effect of group $\left(\mathrm{F}_{(1,64)}=4.95, P=\right.$

$\left.100.03, \eta^{2}=0.07\right)$, but no effect of time $(P=0.73)$ or time $x$ group interaction $(P=$

11 0.45). This difference at the group level suggests that participants were willing to

12 spend more money to sustain goal-directed behavior when the costs of not

13 adhering to this goal were higher. We note also that under these conditions none

14 of the subjects who faced the tempting good consumed it (Figure 3B).

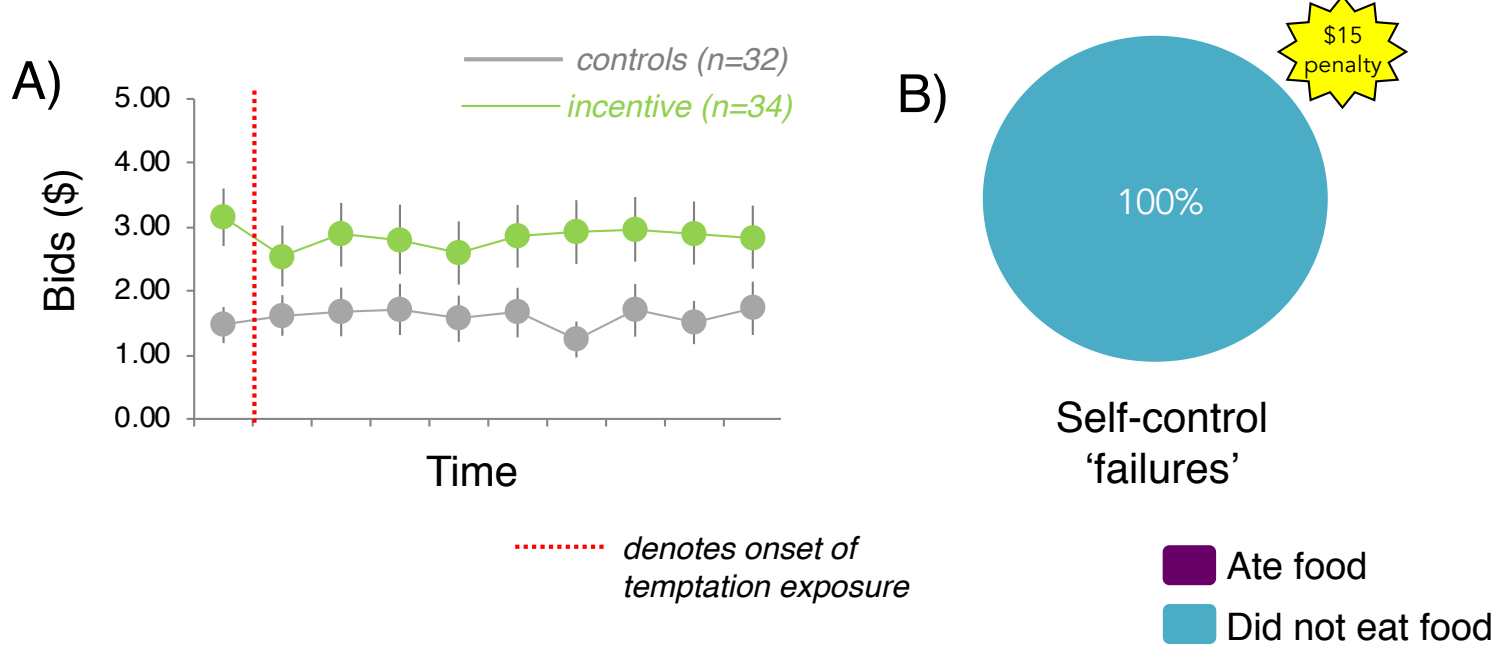

Figure 3. Study 2. (A) Bids to avoid exposure to the tempting food over time in participants for which a $\$ 15$ monetary loss was imposed for consuming the food (depicted in green; Incentive group) and for those where no monetary loss was imposed (depicted in gray; Control group); (B) Proportion of subjects in Study 2 that consumed tempting food during study. 
1 Study 3: Acute stress increases the cost of self-control. Given the tightly

2 coupled relationship between self-control failure and the experience of negative

3 emotional states such as stress, we next examined how exposure to an acute

4 stressor would influence participants' self-control costs. Specifically, we tested

5 the widely held hypothesis that stress makes self-control more 'costly'. To elicit

6 subjective and neurophysiological stress responses, we recruited a new cohort of

7 dieters ( $n=31$ ) that underwent the Cold-Pressor Task (CPT; Lovallo, 1975;

8 Velasco, Gómez, Blanco \& Rodriguez, 1997; McRae et al. 2006) prior to the self-

9 control choice task. The CPT is widely used in laboratory settings to reliably

10 induce mild-to-moderate levels of physiological stress and simply requires

11 participants to submerge their forearms in ice-water continuously for 3-minutes

12 (Methods). Confirming the efficacy of our stress induction procedures,

13 participants in the CPT condition showed elevated concentrations of salivary

14 cortisol, the primary neuroendocrine marker of Hypothalamic-Pituitary-Adrenal

15 (HPA-) axis activity (Figure S1A; SI Results).

16 We assessed whether our stress manipulation influenced the cost of self-control

17 both prior to temptation exposure-when participants were prospectively

18 estimating these costs-and after the highly-tempting food was introduced.

19 Figure 4A depicts aggregate trial-by-trial bidding behavior for participants in the

20 stress condition. A repeated-measures ANOVA across all studies revealed a

21 main effect of group $\left(\mathrm{F}_{(2,94)}=4.4, P=0.01 ; \eta_{\mathrm{p}}^{2}=0.087\right)$, no effect of time $(P=$

$220.45)$ or time $\mathrm{X}$ group interaction $(P=0.64)$. Follow-up t-tests confirmed that

23 stressed participants reported a higher willingness-to-pay overall (mean bid =

$24 \$ 3.38 \pm 3.04 \mathrm{SD}$ ) relative to non-stressed controls (independent samples t-test:

$25 \mathrm{t}_{(61)}=-2.88, P=0.005, \mathrm{~d}=0.72$, two-tailed), suggesting that our experimentally-

26 induced state of stress was reflected in the valuation of pre-commitment to

27 restrict temptation. These increases in the stress group were observed during

28 prospective bids (pre-exposure, $B_{1}{ }_{1}: t_{(61)}=-3.71, P=0.0004, d=0.93$, two-tailed)

29 and persisted across subsequent post-exposure trials (mean of post-exposure 
1 bids: $\left(\mathrm{t}_{(61)}=-2.77, P=0.007, \mathrm{~d}=0.67\right.$, two-tailed). Thus, exposure to acute stress

2 appears to have more than doubled (at a between-subjects level) the average

3 subjective cost of self-control. We note that, similar to Study $1,23 \%$ of our

4 subjects consumed the tempting good (Figure 4B).

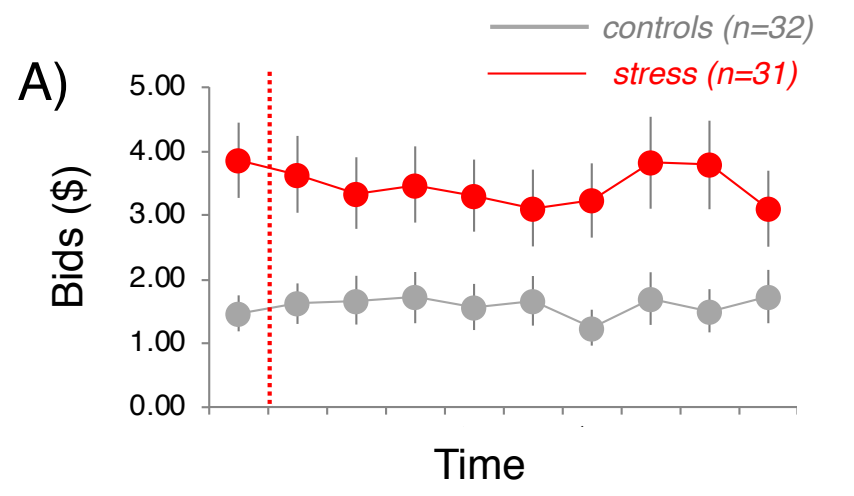

denotes onset of temptation exposure

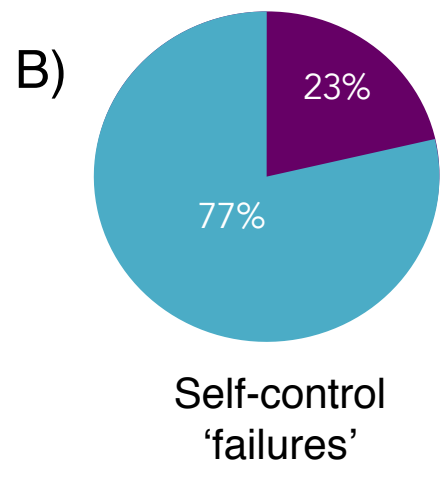

Ate food

Did not eat food

Figure 4. Study 3. (A) Bids to avoid exposure to the tempting food over time for participants that underwent a physiological stress manipulation (depicted in red; Stress group) and for non-stressed participants (depicted in gray; Control group); (B) Proportion of subjects that consumed tempting food during study.

Study 4. The effects of stress and incentives on self-control costs. To assess whether stress exposure increased self-control costs above and beyond that which we observed when motivational incentives were introduced, we

8 conducted an additional study on an independent cohort of dieters. Thirty-one

9 new dieters completed the self-control decision task with the $\$ 15$ penalty for

10 eating the tempting food after undergoing the stress manipulation. We once

11 again observed a reliable willingness-to-pay to avoid temptation (mean bid =

$12 \$ 2.74 \pm 2.20 \mathrm{SD}$ ) that was elevated relative to the Study 1 controls (see below

13 and Figure S2, SI Results; $t_{(61)}=-2.34, P=0.023$, two-tailed). To assess if

14 stress exposure changed bids relative to non-stressed participants who

15 experienced the same incentive structure, we conducted a time $\mathrm{X}$ group RM-

16 ANOVA. However, this analysis revealed no main effect of time $(P=0.55)$ or 
1 group $(P=0.86)$, nor time $\times$ group interaction $(P=0.98)$, thus revealing no

2 significant differences in bidding behavior (Figure 5). We reasoned that a failure

3 to observe group differences as a function of stress exposure could be due to the

4 fact that the stress manipulation in this particular cohort did not effectively elicit

5 cortisol responses. Indeed, an examination of cortisol concentrations revealed

6 that cortisol levels in this cohort did not differ between groups at any time point

7 (Figure S1B, SI Results). Thus, it appears that while motivational incentives led

8 to an increase in bids relative to control participants (replicating our effects from

9 Study 2) we did not observe differences between the two incentive groups as a

10 function of stress exposure, perhaps due to the failure of the stress procedure.

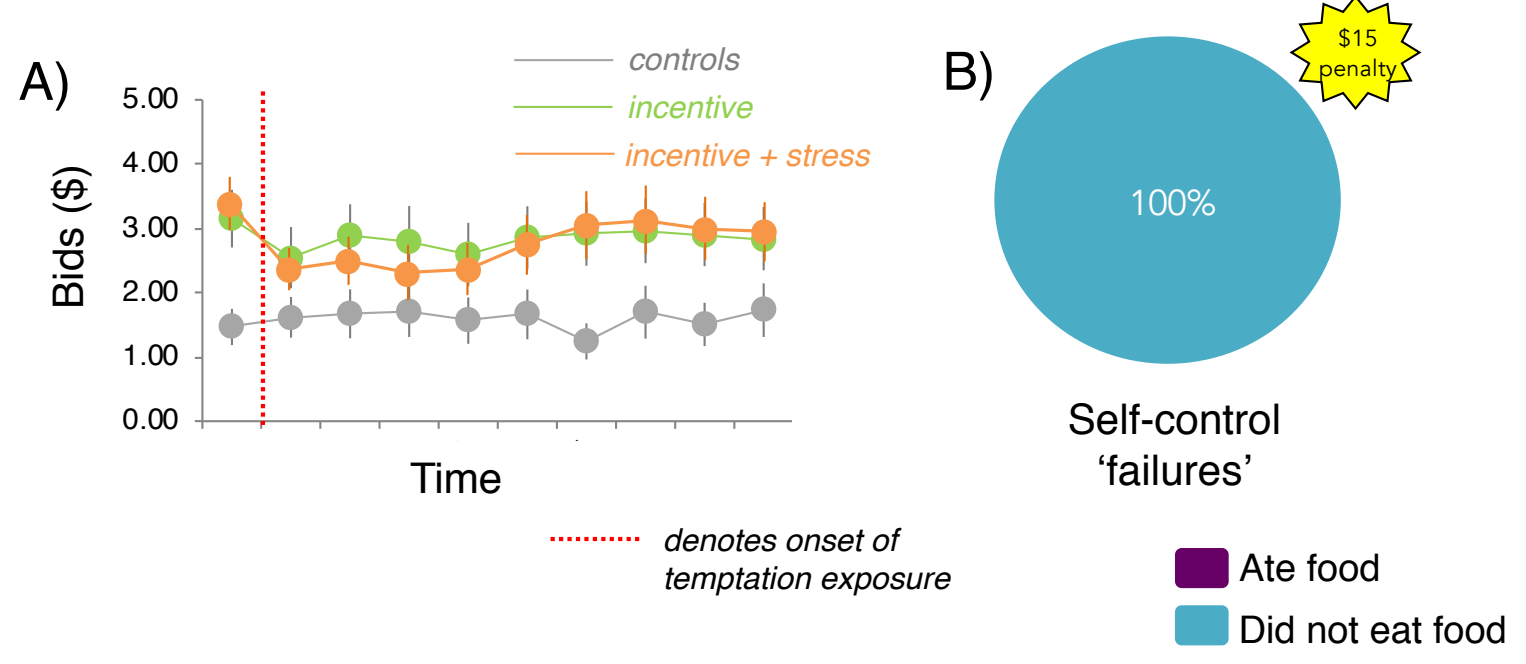

Figure 5. Study 4. (A) Bids to avoid exposure to the tempting food over time in stressed participants for which a $\$ 15$ monetary loss was imposed for consuming the food (depicted in orange; Incentive+Stress group) and for those with the same penalty imposed but no stress induction (depicted in green; Incentive group; Study 2). The control group from Study 1 (no incentive or stress) is depicted in grey for reference. (B) Proportion of subjects from Study 4 that consumed the tempting food during the study. 
1 Secondary Analysis 1: Self-control 'failures' were associated with a higher

2 willingness-to-pay to avoid control. If we assume that subjects do face costs

3 for exercising self-control, then we should expect to see subjects experience self-

4 control failures on occasion. Further, we might expect to find that subjects willing

5 to pay more for pre-commitment experience higher self-control costs and thus

6 might be expected to fail in their self-control more often than subjects who report

7 lower monetary costs for self-control. To test these hypotheses, we examined

8 each subject's behavior after the bidding phase of the experiment was

9 complete-during the final 30-minute phase of the experiment (Methods). During

10 this phase, if no bids had been realized (which was usually the case given the

11 low probability any bid trial was realized), participants were required to remain in

12 the room for the final 30 minutes of the experiment with the tempting food. No

13 further bids were collected during this phase. During this period, we simply

14 observed whether or not each participant consumed the tempting food (a self-

15 control "failure"). The proportion of participants that consumed the tempting food

16 are presented alongside bidding behavior (Figures 2B-5B). We focused our

17 comparison on the study cohorts that shared similar penalty structure in order to

18 control for the increased cost of self-control failure. In Study 1 and 3, where no

19 monetary penalty was imposed for eating the tempting food, $22 \%$ and $23 \%$ of

20 participants consumed the food, respectively (Figure 2B and 4B ). Given that

21 consumption rates did not differ between these two groups, we collapsed across

22 these studies to examine how bidding behavior differed in dieters that ate the

23 food vs. those that did not. Those participants who ate the food were willing-to-

24 pay significantly more to avoid temptation relative to participants who did not eat

25 the food (independent samples t-test: $\mathrm{t}_{(61)}=2.81, \mathrm{P}=0.006$; two-tailed; Figure 6).

26 In Study 2 and 4, where we imposed a monetary penalty for self-control failure,

27 no participants consumed the food (Figure 3B and 5B), thus a comparable

28 analysis to that of above was not possible. We note that the above differences

29 between 'eaters' and 'non-eaters' remain significant even when including all 'non- 
1 eater' participants from Study $3\left(\mathrm{t}_{(95)}=2.41, \mathrm{P}=0.02\right)$ and, additionally, Study 4

$2 \quad\left(t_{(126)}=2.43, P=0.02\right)$.

No incentive groups

(Study 1 \& 3)

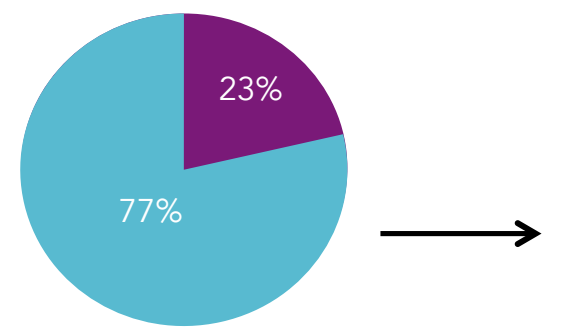

Eat food

Did not eat food

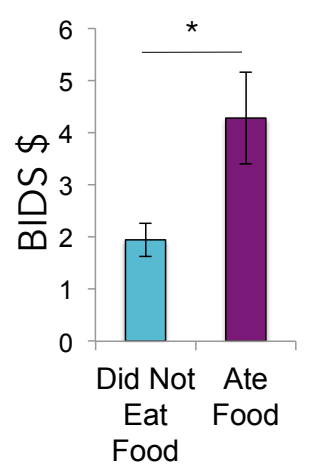

Food
Figure 6. Mean bid for subjects who demonstrated self-control 'failures' (23\%, depicted in purple) and those who did not (77\%; depicted in blue) collapsed across Study 1 (control group) and 3 (stress group). Those participants who consumed the tempting food during the study revealed a higher willingness-to-pay to avoid control. No participants from Study 2 or 4 ( $\$ 15$ penalty groups) consumed the tempting food. Error bars denote SEM.

10 Secondary Analysis 2: Individual difference measures associated with

11 control costs. We next examined how individual differences across our entire

12 sample related to self-control costs. Given that control costs were higher in

13 stressed participants (Study 3), we first sought to characterize how subjectively

14 perceived stress related to willingness-to-pay to avoid self-control. To do this, we

15 correlated mean bids and self-reported stress levels directly before the choice

16 task across participants from all four studies $(n=128)$. Perceived stress was

17 positively correlated with average bidding behavior (Spearman's rho: $r=0.22$,

$18 P=.012$; Figure 7A), suggesting that, across all participants, subjective stress

19 state was related to individuals' willingness-to-pay to avoid temptation. 
Given our evidence that self-control is explicitly costly to choosers, we reasoned that more experience (or success) avoiding temptation might relate to

3 an individual's self-control costs. To explore this question, we conducted a

4 correlation analysis between participants' length of diet and their average

5 willingness-to-pay to avoid exercising self-control. This analysis revealed a

6 significant negative correlation between mean bids and diet length (Spearman's

7 rho: $r=-0.23, P=.01$; Figure 7B). Thus, participants on a diet for a longer length

8 of time tended to pay less to avoid temptation. We note that this does not reveal

9 whether only those with idiosyncratically lower self-control costs succeed at

10 remaining on a diet, whether self-control costs decline as one's diet progresses,

11 or both. However, our method applied longitudinally could be used to answer

12 such questions in future work.
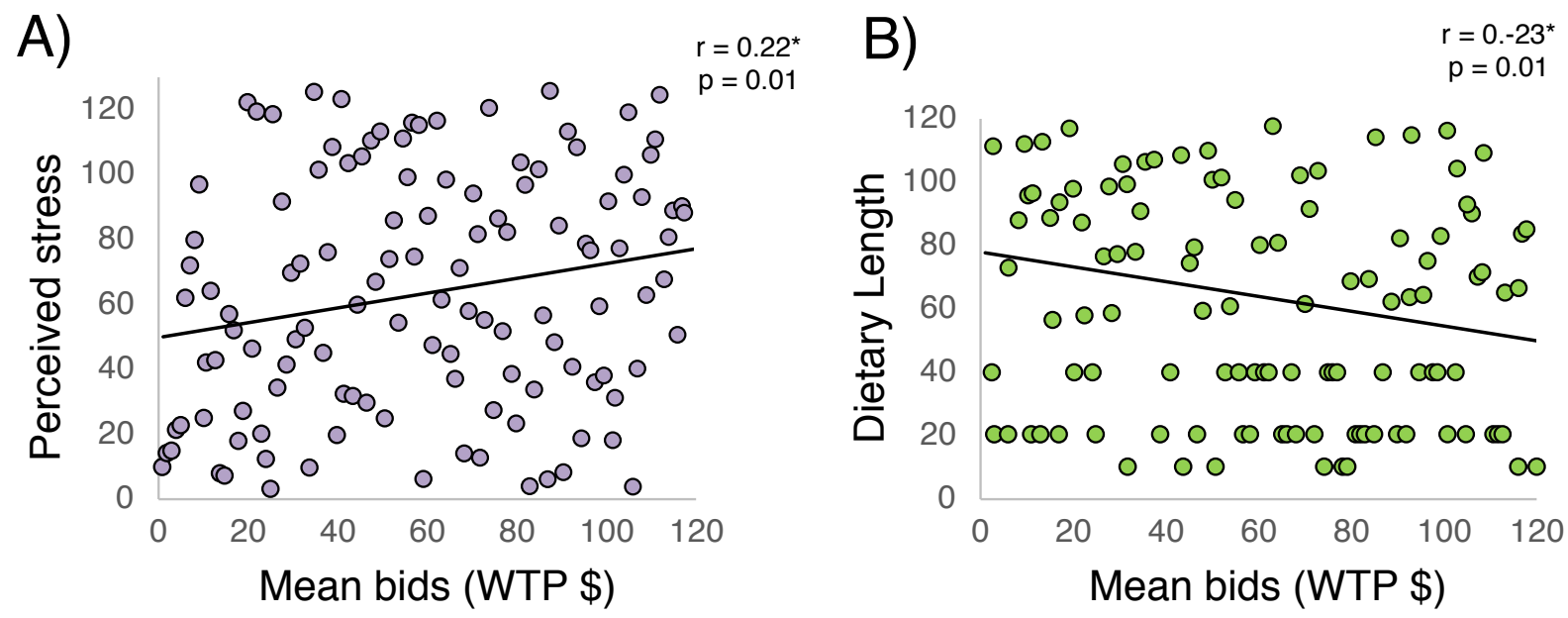

Figure 7. Individual Difference Correlations. (A) Perceived stress was positively correlated with average bidding behavior across participants; (B) Length of diet was negatively correlated with average bidding behavior across participants.

*denotes Spearman ranked correlation

\section{Study 5: Willingness-to-pay to avoid control scales with temptation level. If}

14 participants' willingness-to-pay to avoid temptation does in fact reflect the cost of

15 self-control, then we would expect these costs to scale with varying levels of

16 temptation (i.e., when facing a more highly tempting good, a subject should have

17 to exert more self-control than when facing a less tempting good). To test this, 
1 we conducted a final study in an independent cohort of healthy, hungry dieters. In

2 this study, participants again rated a series of snack foods on how healthy, tasty

3 and tempting they were, which allowed us to identify a low, medium and high

4 tempting food for each individual. On each trial, participants reported their

5 willingness-to-pay (from $\$ 0-\$ 10$, from a $\$ 10$ endowment) to avoid each of the

6 three food items for varying amounts of time (1-60 minutes; Methods). Unlike

7 Study 1-4, all bids were reported prospectively (without any food present) and

8 one trial was randomly selected at the end of the session to be realized.

$9 \quad$ Figure 8 depicts average bids for each time point for each level of

10 temptation. A temptation level (low, medium, high) $\mathrm{X}$ time (1-60 minutes) RM-

11 ANOVA revealed a significant main effect of temptation level $\left(F_{(2,30)}=33.06, P<\right.$

$\left.12.0001, \eta_{p}^{2}=0.69\right)$ and time with food $\left(F_{(9,135)}=29.12, P<.0001, \eta_{p}^{2}=0.67\right)$, as

13 well as a temptation $X$ time interaction $\left(F_{(18,270)}=5.75, P<.0001, \eta_{p}{ }^{2}=0.27\right)$.

14 Bids differed significantly for foods with low (mean bid $=\$ 1.16 \pm 0.36 \mathrm{SD}$ ),

15 medium (mean bid $=\$ 2.99 \pm 1.02 \mathrm{SD}$ ), and high (mean bid $=\$ 4.96 \pm 1.30 \mathrm{SD}$ )

16 temptation levels. Further, bids scaled with each temptation level across

17 increasing amounts of time (Figure 8). Planned contrasts demonstrated a

18 significant linear increase in bids with higher temptation level $\left(F_{(1,15)}=43.95, P<\right.$

$19.0001)$ and, separately, with increased time with food $\left(F_{(1,15)}=44.81, P<.0001\right)$.

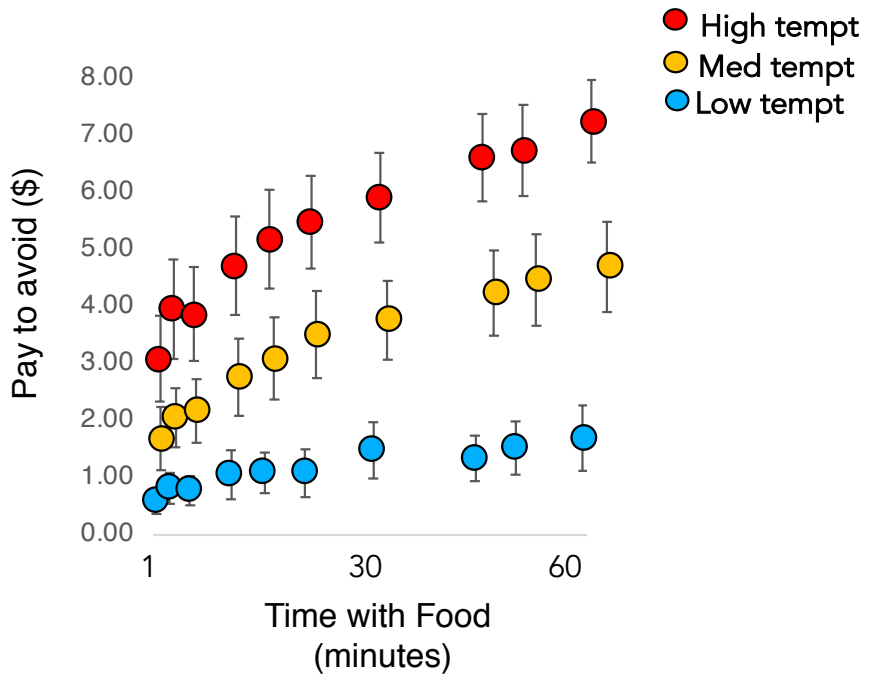

Figure 8. Willingness-to-pay to avoid foods that varied in temptation level and amount of time required to spend with food. Bids scaled with increasing time with and temptation of the food. Error bars depict SEM. 


\section{DISCUSSION}

2 A universal paradox in human behavior is our tendency to set difficult long-term

3 goals but then to make choices that appear to contradict or undermine those

4 goals. Using an economic decision-making paradigm, coupled with a sample of

5 dieters avoiding tempting food rewards as a model of self-control more broadly,

6 we found that people will pay to avoid temptation, quantitatively revealing their

7 subjective cost of control under a variety of circumstances. We found that these

8 costs are modulated by incentives (shows cost sensitivity; Study 2/4) and acute

9 stress exposure (Study 3), consistent with the notion that motivational and

10 affective state modulate one's willingness to exercise self-control. In a final study

11 designed to test how self-control costs scale with increasing levels of temptation

12 (Study 5), we showed that longer exposure to a tempting good imposes higher

13 self-control costs (self-control costs obey monotonicity) and more tempting goods

14 impose higher control costs at the within-subject level than do lower tempting

15 goods (self-control costs order with temptation).

17 Decades of psychological research have revealed that the act of engaging in

18 self-control is subjectively effortful and aversive. Emerging work in the cognitive

19 control literature has proposed that this experience stems from the cognitive

20 costs imposed by deploying control, an account consistent with more recent

21 economic theories of self-control (Gul \& Pesendorfer, 2001/2004) that propose

22 preferences for pre-commitment reveal an inherent psychological cost to

23 resisting temptation. These converging lines of work provide a clear and testable

24 hypothesis: Self-control failures may be conceptualized as a rational decision

25 that emerges when the costs of exercising control exceed the relative perceived

26 benefits (Kurban et al., 2013; Berkman et al., 2017; Kool \& Botnivick, 2018).

27 However, without a psychophysical method to precisely quantify these costs,

28 researchers often must infer whether and how much self-control a chooser

29 requires to make goal-consistent decisions or to delay gratification. Our findings

30 unite psychological and economic theories of self-control and provide empirical 
1 evidence that self-control is explicitly costly to humans and that these costs can

2 be quantified by measuring the value of pre-commitment to restrict temptation.

4 Gul and Pesendorfer's theory is consistent with a growing body of psychological

5 and neuroscience research that suggests people view cognitive demand as

6 intrinsically costly and tend to avoid utilizing cognitive resources if possible

7 (Kurzban et al. 2013; Westbrook \& Braver, 2015; Shenhav et al. 2013/2017; Kool

8 \& Botnivik, 2018). These costs are thought to stem from limitations in the

9 cognitive resources available to support the demands of control, suggesting self-

10 control arises from evaluations of how valuable expenditures of control are

11 perceived to be relative to how costly. This is a notable departure from classic

12 psychological self-control models that view cognitive resources as depletable

13 (i.e., both limited and diminished with use), arguing instead that such resources

14 are finite and reallocated dynamically depending on the perceived costs and

15 benefits (Kurzban et al., 2013; McGuire \& Kable, 2015). Why some classes of

16 cognitive control may be aversive or costly remains unclear. However, the

17 approach presented here offers a metric for how aversive or costly individuals'

18 find the exertion of self-control to be on a moment-to-moment basis.

20 Our task employed two important features to probe the nature of self-control.

21 First, our task measured momentary willingness to pay to avoid control

22 prospectively (prior to food exposure) and again after participants encountered

23 the tempting food. One possible explanation for why self-control appears to fail

24 so often is that individuals may poorly estimate how costly self-control will be.

25 Our data tends to lean against this conclusion, at least in this particular choice

26 setting. We observed no significant difference in measured control costs before

27 versus after food exposure. This suggests that our participants had an accurate

28 prospective awareness of the self-control costs they would later face. A second

29 important feature of our task was that-unlike existing self-control decision

30 paradigms-our bidding measurements were collected continuously over time, 
1 allowing us to track how these costs change with continued exposure to

2 temptation. Some existing work (Baumeister, Bratslavsky, Muraven, \& Tice,

3 1998; Muraven \& Baumeister, 2000) suggests that that self-control becomes

4 more difficult as it is continuously exerted. If this were true for our participants,

5 we would expect their self-control costs to increase as the experiment

6 progressed. Interestingly, we instead observed that participants' bids to avoid

7 control were quite stable over time. The fact that participants' control costs did

8 not increase over time, however, is consistent with a growing body of work

9 suggesting that performance reductions are a not mandatory feature of engaging

10 in control (Kurzban et al., 2013; Shenhav et al. 2013/2017; Kool \& Botnivik,

11 2018). Our findings are consistent with value-based frameworks that argue

12 individuals need not necessarily experience decrements in control performance

13 as long as the perceived benefit of deploying control continues to outweigh the

14 cost. We note, however, that our data on this point is relevant only to the

15 intervals of time tested here (under an hour in total duration).

17 The stability in our participants' willingness to pay to avoid exerting control may

18 also reflect the lack of temporal uncertainty inherent in our task. In a recent line

19 of work, McGuire and Kable (2013/2015) demonstrated that behavior in a range

20 of delay-of-gratification tasks (including the 'marshmallow task', Mischel \&

21 Ebbesen, 1970) might be explained by the underlying predictions participants

22 have regarding when a delayed outcome will arrive. They suggested that one

23 major reason individuals appear to 'succumb' to temptation is that under

24 temporal uncertainty, individuals may rationally conclude that the delayed

25 outcome may no longer be worth waiting for. In our study participants were fully

26 informed regarding the temporal structure of the task and were at all times aware

27 that self-control would be engaged only for a finite period of time. This feature of

28 our task may explain the stability observed in participants reported bids over

29 time. An open question for future research is whether imposing temporal

30 uncertainty, or requiring self-control for longer periods of time (Blain et al., 2016) 
1 than we used in our task, might lead to an increase in ongoing self-control costs

2 even at this limited time interval.

4 Self-control research across disciplines suggests that we should be able to

5 induce changes in these costs with changes in motivational state. Consistent with

6 the notion that decisions to engage in self-control arise from a dynamic cost-

7 benefit evaluation (Berkman et al., 2016), we found that willingness to pay for

8 pre-commitment increased as the cost of failing to adhere to one's diet

9 increased. When faced with losing \$15 in addition to failing to adhere to their diet,

10 participants were willing to pay to restrict access to temptation, thus pre-

11 commitment became more valuable (Study 2 and 4). These findings are

12 consistent with work showing that motivational incentives can increase

13 willingness to engage in self-control strategies (Hagger et al, 2010; Krebs et al.,

14 2010) and demonstrate overall cost-sensitivity in the self-control mechanism.

16 We also observed an increase in the cost of self-control when participants were

17 successfully stressed using an acute stress induction (Study 3). Stress exposure

18 has long been thought to compromise self-control (Hockney, 1983; Holding,

19 1983), and this intuition has been borne out in a large body of empirical work-

20 from the cognitive neuroscience literature that shows stress diminishes cognitive

21 capacity and flexibility (Schoofs et al 2009; Plessow et al 2009) and selectively

22 reduces goal-directed control of decisions (Schwabe \& Wolf 2009; Otto, Raio, et

23 al., 2013) to the clinical literature where stressors remain a primary risk factor for

24 the emergence and relapse of addiction-related disorders (Sinha, 2011). Our

25 findings provide a direct test of whether stress compromises self-control by

26 increasing its associated cognitive cost. This relationship was also observed

27 beyond participants assigned stress condition, as higher self-control costs were

28 positively associated with perceived stress scores. We note that in Study 4,

29 where both stress and incentives were imposed before the self-control task, we

30 did not observe an added increase in bids above and beyond that of the Study 2 
1 incentive group. However, this may be due to the fact that we did not see

2 evidence of a cortisol elicitation from the stress manipulation. Future work may

3 seek to use alternate stress induction techniques (e.g., social or cognitive

4 stressors) to test if other types of stressors lead to additive (or interactive) effects

5 with motivational incentives on self-control costs. Finally, one can imagine

6 decision environments whereby stress can impair precommitment decisions

7 given their reliance on prospective thinking. Our task utilized both prospective

8 and experiential exposure to temptation but it would be interesting to examine

9 how stress affects precommitment decisions that rely fully on future prospection

10 or memory retrieval.

12 Finally, in Study 5, confirming that our bids did in fact reflect the cost of self-

13 control and not random baseline bidding behavior, we found that the average

14 magnitude of bids scaled with increasing levels of temptation. By testing bidding

15 behavior across varying degrees of temptation level and a broader range of times

16 with the food, we were able to confirm that willingness to pay for pre-commitment

17 tracks with the increased cost of resisting temptation, demonstrating that the cost

18 of self-control appears to increase monotonically with duration and that more

19 highly tempting goods induce higher self-control costs. Overall, our findings

20 suggest that measuring the control costs can reveal unique information about the

21 subjective experience of resisting temptation that cannot be attained using

22 existing measures of self-control.

24 Given its importance as a strategy to help individuals achieve their long-term

25 goals by reducing self-control costs, a number of recent studies have begun to

26 measure preferences for pre-commitment in the laboratory (Crockett et al., 2013;

27 Schwartz et al., 2014; Soutschek et al., 2017/2020; Studer et al., 2019). For

28 example, Crockett et al. (2013) provided the first empirical demonstration that

29 pre-commitment facilitates choices for larger, later rewards as opposed to

30 smaller, sooner ones when explicitly offered as a choice strategy in an 
1 intertemporal choice task (e.g. viewing erotic images that varied in reward

2 magnitude and delay). This study further demonstrated that the primary neural

3 circuits underlying pre-commitment (e.g., lateral frontopolar cortex; also see

4 Soutschek et al., 2017) are distinct from that of standard self-control ("willpower")

5 tasks that rely on the effortful inhibition of impulses (e.g., dorsolateral PFC,

6 inferior frontal gyrus), consistent with the notion these self-control strategies rely

7 on different neurocognitive processes. These data, coupled with more recent

8 work showing that higher impulsivity and meta-cognitive awareness leads to

9 stronger preferences for pre-commitment (Soutschek et al., 2020), are consistent

10 with the view that pre-commitment decisions engage future planning and

11 prospection and are driven by an awareness of subjective self-control costs.

13 Our results add to this growing literature by demonstrating that individuals not

14 only show preferences for pre-commitment, but they are willing to pay to adopt

15 such strategies, effectively revealing their subjective cost of self-control over time

16 and with continued exposure to temptation. Our task extends extant studies of

17 pre-commitment outside of classic intertemporal and effort-based choice

18 paradigms by directly quantifying the cost of resisting temptation under different

19 motivational/affective states and levels of temptation. One relevant question for

20 future work is how we can further dissociate the cost-benefit processes that drive

21 pre-commitment decisions. For example, future work might address to what

22 extent individuals pay to remove temptation in order to avoid a self-control failure,

23 versus to diminish the disutility of resisting temptation irrespective of predicted

24 failures. One recent study, for example, found that pre-commitment increases

25 motivation to engage in effortful action that leads to larger rewards. Specifically,

26 in the context of an effort- and delay-based choice task, precommitment choices

27 were found to be driven by a desire to reduce opportunity costs and secure

28 adequate motivation to endure the longer delay or higher effort required to attain

29 larger rewards, rather than to avoid a failure of willpower per se (Studer et al.,

30 2019). Future work may attempt to further dissociate the motivational processes 
1 that underlie these decisions and how changes in temptation intensity or

2 temporal uncertainty may alter them.

4 A number of limitations should be noted for future work. First, we acknowledge

5 that there are likely many different components to what makes the exertion of

6 self-control cognitively costly. For example, there may be cognitive costs to

7 resisting temptation and also personal and health costs associated with self-

8 control failures. While our task does not currently dissociate among the

9 components that feed into self-control costs more generally, this is an open area

10 for future research. Second, unlike some studies in the human stress literature

11 we included both men and women in each of our samples. However, we did not

12 measure menstrual phase, oral contraceptive use or cycle-dependent sex

13 hormones, which have been shown to impact stress responses in women

14 (Kirschbaum et al., 1999; Hellhammer et al., 2009). Future work measuring such

15 factors may reveal interesting patterns in control costs that we were unable to

16 detect here. Finally, despite every effort to eliminate them from our procedure (SI

17 Materials), we cannot definitively rule out the possibility that demand

18 characteristics may have contributed to these effects in some way.

20 In summary, we report a novel task for measuring the subjective cost of self-

21 control. Our findings are consistent with emerging work across disciplines

22 suggesting that self-control and its failures can be seen as fundamentally rational

23 responses to a complex world in which individuals' trade-off the cognitive cost of

24 resisting immediate temptation against the benefits of achieving future goals. 


\section{METHODS}

2 Participants. 138 healthy young adult participants that indicated they were on a

3 diet to maintain or lose weight participated in the study (see SI Materials for full

4 screening criteria). Participants were recruited using flyers posted on and around

5 the NYU campus, as well as electronic advertisements on New York University's

6 Department of Psychology website. Participants were excluded prior to

7 participation for the following reasons: (1) pregnancy; (2) high-blood pressure or

8 a heart condition; (3) history or medication for neurological or psychiatric

9 disorders; (4) diabetes, metabolic disorders, food allergies or history of eating

10 disorders; and (5) use of corticosteroids or beta-blockers. All participants

11 provided written informed consent in accordance with experimental procedures

12 approved by the New York University Committee on Activities Involving Human

13 Subjects. All research and experimental procedures were performed in

14 accordance with approved IRB guidelines and regulations.

15 Subjects were paid $\$ 15$ per hour plus a $\$ 10$ bidding endowment. Six participants

16 from the stress groups were unable to complete the CPT task and were thus

17 excluded. Two additional participants were removed prior to data analysis

18 because they revealed that they were on special diets to sustain (and ideally

19 increase rather than decrease) weight and two others were excluded for being on

20 medication (revealed after the experiment ended). Our final analysis included a

21 total of 128 healthy participants (84 women) with a mean age of 24.37 (SD =

227.07 ; range $=18-55$ ).

24 General Procedure (Study 1-4). Hungry, healthy dieters were asked to abstain

25 from eating 3-4 hours before participating in the study. Upon arrival at the

26 laboratory, participants provided informed consent and were escorted to the

27 experiment room for a 10-minute acclimation period, after which they rated their

28 current hunger level (from 1-10; Fig. S3 and S4, SI Results), completed the food

29 rating and ranking scales (Fig. S5, SI Results) and provided basic information 
1 about their current diets (Fig. S6, SI Materials). After baseline cortisol was

2 collected, participants received their $\$ 10$ (cash) endowment. They then received

3 instructions regarding the self-control decision task and were explicitly informed

4 about which high- and low-tempting foods they would be making choices about

5 during the study. (Participants in Studies $2 \& 4$ were further instructed that they

6 would lose a $\$ 15$ bonus payment provided at the end of the study if they

7 consumed the tempting food at any point.) All participants then completed either

8 the CPT or control task and were given a 10-minute break in the experiment

9 room before an additional cortisol sample was collected. This break was

10 implemented to ensure that cortisol levels induced by the CPT would begin to

11 peak in coordination with the choice task. Participants then completed the self-

12 control decision task (see Decision-Making Task), during which they indicated

13 the maximum amount that they would be willing to pay to remove the high-

14 tempting food from the room and replace it with the low-tempting food. After the

15 30-minute bidding phase of the task was complete, the final cortisol sample was

16 collected and the final phase of the experiment began, during which participants

17 were required to remain in the experiment room with the high- or low-tempting

18 food for the final 30 minutes of the study (see Bid Realization). Once this 30-

19 minute final phase was over participants were paid for their time and left the

20 laboratory.

22 Stress Induction Technique. All participants in the stress group (Studies 3 \& 4)

23 completed the CPT, for which participants submerged their right forearms in ice-

24 cold water $\left(0^{\circ} \mathrm{C}\right.$ to $\left.4^{\circ} \mathrm{C}\right)$ for 3 min continuously. All participants in the control

25 group (Studies $1 \&$ 2) followed the exact same procedure using room-

26 temperature water $\left(30^{\circ} \mathrm{C}\right.$ to $\left.35^{\circ} \mathrm{C}\right)$. The $\mathrm{CPT}$ is widely used in laboratory

27 settings to model the effects of mild to moderate stress and reliably generates

28 both autonomic nervous system and HPA-axis activation, as measured by

29 increased physiological arousal, neuroendocrine responses, and subjective

30 stress ratings (Lovallo, 1975; Velasco, Gómez, Blanco \& Rodriguez, 1997; 
1 McRae et al. 2006).

3 Neuroendocrine Assessment. Saliva samples were collected throughout the

4 study to assess cortisol concentrations, which serve as neuroendocrine markers

5 of stress response. Participants were run between 12 and $5 \mathrm{pm}$ to control for

6 diurnal rhythms of stress hormone levels. Saliva samples were collected using a

7 high-quality synthetic polymer-based salivette placed under participants' tongues

8 for two minutes. Participants were initially seated in the experiment room for a

9 10-minutes acclimation period, during which they drank 4 oz of water to clear any

10 residual saliva. Samples were collected at baseline (sample 1), 10 minutes after

11 the CPT/control task administration (sample 2), and directly before the choice

12 realization began ( 30 minutes after the CPT/control task administration; sample

13 3). Samples were immediately stored in a sterile tube and preserved in a freezer

14 set to $-80^{\circ} \mathrm{C}$. Samples were analyzed by the Psychobiological Research

15 Laboratory of the University of Trier, Germany, using a time-resolved

16 immunoassay with fluorometric detection (DELFIA, cf. Dressendörfer et al.,

17 1992). Duplicate assays were conducted for each sample, and the average of the

18 two values was used in our analyses. Any samples that contained insufficient

19 saliva could not be analyzed and were excluded from our analyses. Cortisol data

20 was log-transformed to account for the skewed nature of cortisol distributions

21 (Fig. S1A and S1B, SI Results).

23 Food Item Scales \& Selection. In order to select a high- and low-tempting food

24 item for each individual, participants completed a series of food rating scales

25 prior to the study (Fig. S5, SI Results). Participants separately rated 20 food

26 items (Fig. S7, SI Materials) on how tasty, healthy and tempting these items

27 were from 1 (not at all) to 10 (very much so). Participants then ranked these 20

28 food items from best (\#1) to worst (\#20) for their current diet. Low-tempting foods

29 were chosen by a computer algorithm that identified foods that fell in the lowest

$3020 \%$ of taste and temptation ratings, the highest $20 \%$ of health ratings and that 
1 was ranked in the upper $10 \%$ of foods best for the participants' current diet.

2 Conversely, high-tempting foods were identified as those that fell in the upper

$320 \%$ of taste and temptation ratings, the lowest $20 \%$ of health ratings and that

4 was ranked in the lowest $10 \%$ of foods worst for the participants' current diet.

6 Decision-making Task (Figure 1). To directly examine individuals' subjective

7 cost of self-control, we designed an incentive-compatible decision-making task

8 that measured the monetary costs that participants were willing to incur to avoid

9 temptation on a moment-to-moment basis. There were two phases of the task:

10 (1) a bidding phase, during which participants indicated the maximum they would

11 be willing-to-pay from a $\$ 10$ endowment to remove the high-tempting food from

12 the room and replace it with the low-tempting food; and (2) a final realization

13 phase, during which participants sat in the experiment room with the food for the

14 final 30 minutes of the experiment. Participants in Studies $2 \& 4$ were further

15 instructed that they would lose a $\$ 15$ bonus payment at the end of the study if

16 they consumed the tempting food at any point.

17 On each trial, participants viewed a computer screen that prompted them

18 to enter the maximum amount that they were currently willing-to-pay to remove

19 the high-tempting food from the room and replace it with the low-tempting food.

20 Participants registered their bids with an open response-window by using the

21 mouse to control a sliding bar that ranged from $\$ 0$ to $\$ 10$ (in $\$ 0.01$ increments)

22 and clicking the mouse on their selected bid. Bid trials were presented

23 approximately every 3 minutes (Studies $2 \& 4$ ) or 2 minutes (in an effort to

24 acquire more precise temporal measurements of bids) for a total of 10 bids for

25 Study $2 \& 4$ and 15 bids for Studies $1 \& 3$. Choices were presented using

26 PsychToolBox.

27 After each bid was received there was a $2 \%$ chance that this bid would be

28 immediately employed in a procedure that would lead to the final 30-minute

29 phase in which the high-tempting or low-tempting food would be in the room with

30 the subject. This incentivized participants to bid their true value for removing the 
1 high-tempting food since the bidding phase of the task could end on any trial and

2 only the current bid would be used to determine whether the food was removed

3 for the 30 -minute final phase. The $2 \%$ hazard rate also ensured that the majority

4 of bidding trials would not be realized, allowing us to track the dynamics of how

5 self-control costs change over time with greater exposure to temptation. Finally,

6 this feature of the task allowed us to eliminate any effect of temporal discounting

7 on the sequential bids. To realize bids, we used a standard Becker-DeGroot-

8 Marschak (BDM) auction procedure widely used to reveal maximum willingness-

9 to-pay (see Bid Realization Procedure below).

10 For the initial bidding trial, no food was present in the room. If this initial

11 trial was not realized, the food was brought in the room and remained there until

12 a bid was implemented or the bidding phase concluded. (If the trial was realized

13 the BDM procedure determined whether the food was brought into the room for

1430 minutes. Immediately after each bidding trial, participants were notified as to

15 whether that particular bid would be implemented. If no bid was realized by the

16 end of the 30-minute bidding phase, the task transitioned automatically to the

17 final 30-minute realization phase.

19 Self-Control Failures. Any quantity of food consumed during the task was

20 considered a self-control failure. In all cases of eating, participants consumed the

21 entire item with the exception of one participant who consumed half of the snack

22 food (potato chips) presented. Any participants that consumed the food did so

23 during the final 30-minute realization phase. To reduce observer effects,

24 participants were alone in the experiment room for the duration of the study so

25 we were not present to measure the precise time at which they might have eaten

26 the tempting food. Whether or not they consumed the food was revealed after

27 participants left the laboratory.

28 Bid Realization Procedure. To determine whether the participant won or lost

29 the opportunity to replace the high-tempting food with a low-tempting food, a 
1 standard economic auction procedure was implemented (DeGroot-Marschak;

2 BDM). Participants selected a selling chip from a bag (chips ranged from $\$ 0$ to

$3 \$ 10$ in $\$ 0.01$ increments) and this selected chip represented the winning sell

4 price. This randomly selected sell price was then compared against the

5 participant's current bid. If the bid price was greater or equal to that of the sell

6 price, then the participant won the auction and they paid the sell price (not the bid

7 price) from their endowment to have the high-tempting food removed. If the bid

8 was lower than the sell price the participant lost the auction. In this case, the

9 high-tempting food remained in the room for the remainder of the experiment and

10 the participant would keep the entire endowment. This procedure incentivizes

11 participants to report their true maximum willingness-to-pay.

13 Study 5. An additional 20 participants were recruited following the same

14 recruitment, screening, informed consent and payment procedures as Study 1-4

15 (Participants). Two participants were excluded for using medications on our

16 exclusion criteria list. Due to a computer software error, data from two other

17 participants was not recorded. Participants were asked to refrain from eating

18 prior to coming into the lab and began by rating the same series of 20 food items

19 (see Figure $\mathbf{S 7}$ for choice set) on how tasty, healthy and tempting these items

20 were from 1 (not at all) to 10 (very much so). These ratings allowed us to select a

21 low, medium and high tempting food for each participant. On each trial,

22 participants viewed an image of a snack food that varied on temptation level (low,

23 moderate, high) and amount of time for which participants would potentially have

24 to spend with the food (1, 3, 5, 10, 15, 20, 25, 30, 45, 60 minutes). Participants

25 viewed the food for 4 seconds and the entered their how much they were willing

26 to pay (from \$0-\$10) from a $\$ 10$ endowment to avoid that food, given the

27 temptation level, quantity and time amount. After the 90 trials were completed,

28 one trial was randomly selected and the same BDM auction procedure was used

29 to identify whether the participants won or lost the auction based on their bid for

30 that given trial (Bid Realization Procedure). All participants remained in the 
1 experimental room for 1 hour after the bidding task was complete in order to

2 control for the cost of time (i.e., to ensure bids did not reflect an aversion to

3 waiting for the allocated amount of time relative to the cost of control). If

4 participants lost the auction, the food was present in the room for the amount of

5 time stated on the selected trial (e.g., if the trial depicted 15 minutes with the

6 food, then the participant spent 15 minutes of the full hour with the food). If they

7 won the auction then the food was not present during this amount of time.

9 Data Analysis. All statistical analysis for behavioral and cortisol data was carried

10 out using SPSS (version 20.0, 2011; IBM Corp., Armonk, NY). Due to the

11 skewed nature of cortisol concentration distributions documented in the literature

12 (Miller et all, 2013), cortisol values were log-transformed in order to better

13 approximate a Gaussian (normal) distribution. Data were tested for equal

14 variances using Mauchly's sphericity tests and Greenhouse-Geisser corrections

15 were performed to address any violations of sphericity. Analysis of variance

16 (ANOVA) with repeated measures was used to analyze all choice (i.e., bidding)

17 and cortisol data. Post hoc comparisons were conducted using Student $t$-tests

18 when appropriate. All tests were two-tailed and considered statistically significant

19 when $\mathrm{p}<.05$.

20 Data and Code Availability. The data sets generated during and/or analyzed

21 during the current study are available from the corresponding author on

22 reasonable request.

Acknowledgments. We thank all members of PWG's laboratory, especially Anna Konova, Alexandra Mellis and Christopher K. Steverson, for helpful feedback, comments and discussion. This work was supported by NIH Grant F32MH110135 and a NARSAD Young Investigator Grant from the Brain \& Behavior Research Foundation to CMR and NIH Grant R01DA038063 to PG. 


\section{References}

Ainslie, G. (1975) Specious reward: a behavioral theory of impulsiveness and impulse control. Psychological Bulletin, 82 (4), 463-496.

Ariely, D. \& Wertenbroch, K. (2002) Procrastination, deadlines, and performance: self-control by precommitment. Psychological Sciences, 13(3): 219-24.

Arnsten AFT (2009) Stress signalling pathways that impair prefrontal cortex structure and function. Nat Rev Neurosci 10(6):410-422.

Becker GM, DeGroot MH, Marschak J (1964) Measuring utility by a singleresponse sequential method. Behavioral Science, 9(3): 226-32.

Berkman, E.T., Hutcherson, C.A., Livingston, J.L., Kahn, L.E. and Inzlicht, M. (2017) Self-control as a value-based choice. Current Directions in Psychological Science, 26(5):422-428.

Baumeister, R. F., \& Heatherton, T. F. (1996). Self-regulation failure: An overview. Psychological Inquiry, 7, 1-15.

Baumeister, R. F., Bratslavsky, E., Muraven, M., \& Tice, D. M. (1998). Ego depletion: Is the active self a limited resource? Journal of Personality and Social Psychology, 74, 1252-1265.

Bastien, B., Hollard, G., and Pessiglione, M. (2016) Neural fatigue and intertemporal choice. Proceedings of the National Academy of Sciences, 113 (25) 6967-6972.

Bryan, G., Karlan, D. and Nelson, S. (2010) Commitment Devices. Annual Review of Economics, 2, 671-98.

Crockett, M.J., Braams B.R., Clark, L., Tobler, P.T., Robbins, T.W. and Kalenscher, T. Restricting temptations: neural mechanisms of precommitment. Neuron, 79(2), 391-401.

Fishburn, P.C., Rubinstein, A., 1982. Time preference. Int. Econ. Rev. 23 (3), 677694.

Frederick, S., Loewenstein, G., O'Donoghue, T., 2002. Time discounting and time preference: a critical review. Journal of Economic Literature, 40, 351401.

Fudenberg, D., \& Levine, D.K. (2006) A dual-self model of impulse control." American Economic Review, 96(5): 1449-1476. 
Gul, F. \& Pesendorfer, W. (2001) Temptation and self-control. Econometrica 69(6):1403-35.

Gul, F. \& Pesendorfer, W. (2004) Self-control and the theory of consumption. Econometrica 72(1):119-58.

Hagger, M. S. et al. A multilab preregistered replication of the ego-depletion effect. Perspect. Psychol. Sci. 11, 546-573 (2016).

Hellhammer, D.H., Wu"st, S. and Kudielkam B.M. (2009) Salivary cortisol as a biomarker in stress research. Psychoneuroendocrinology, 34, 163-171.

Hockey, G. (1s983) Stress and fatigue in human performance. Wiley. [MI]

Holding, D. H. (1983) Fatigue. In: Stress and fatigue in human performance, ed. G. Hockey, pp. 145-67. Wiley.

Kable, J.W., and Glimcher, P.W. (2007). The neural correlates of subjective value during intertemporal choice. Nature Neuroscience, 10, 1625-1633.

Kahneman, D. (2011). Thinking, fast and slow. London: Allen Lane.

Kirschbaum, C., Kudielka, B.M., Gaab, J., Schommer, N.C., Hellhammer, D.H. (1999) Impact of gender, menstrual cycle phase, and oral contraceptives on the activity of the hypothalamus-pituitary-adrenal axis. Psychosom Med. 61:154-162.

Kool, W., \& Botvinick, M. (2018) Mental labour. Nature Human Behavior, 2: 899908.

Krebs, R. M., Boehler, C. N. \& Woldorff, M. G. (2010) The influence of reward associations on conflict processing in the Stroop task. Cognition 117(3):341-47.

Kurzban, R., Duckworth, A., Kable, J.W. and Myers, J. (2013) An opportunity cost model of subjective effort and task performance. Behavioral and Brain Science, 36, 661-679.

Laibson D. Golden eggs and hyperbolic discounting. Q J Econ 1997;12:443-477

Lovallo W (1975) The cold pressor test and autonomic function: A review and integration. Psychophysiology 12:268-282.

Mazur, 1982McRae AL, et al. (2006) Stress reactivity: Biological and subjective responses to the cold pressor and Trier Social stressors. Hum Psychopharmacol 21(6):377-385. 
Mischel, W., \& Ebbesen, E. B. (1970). Attention in delay of gratification. Journal of Personality and Social Psychology, 16, 329-337.

Mischel, W., Ebbesen, E. B., \& Zeiss, A. R. (1972). Cognitive and attentional mechanisms in delay of gratification. Journal of Personality and Social Psychology, 21, 204-218.

Mischel, W., Ayduk, O., \& Mendoza-Denton, R. (2003). Sustaining delay of gratification over time: A hot-cool systems perspective. In G. Loewenstein, D. Read, \& R. F. Baumeister (Eds.), Time and decision: Economic and psychological perspectives on intertemporal choice (pp. 175-200). New York, NY: Sage.

Mischel, W., Shoda, Y., and Rodriguez, M.I. (1989). Delay of gratification in children. Science, 244, 933-938.

McGuire, J.T. and Kable, J.W. (2013) Rational temporal predictions can underlie apparent failures to delay gratification. Psychological Review, 120(2), 395-410.

McGuire, J.T. and Kable, J.W. (2015) Medial prefrontal cortical activity reflects dynamic re-evaluation during voluntary persistence. Nat Neurosci 18, 760-766.

Miller, R., Plessow, F., Rauh, M., Gröschl, M., and Kirschbaum, C. (2013) Comparison of salivary cortisol as measured by different immunoassays and tandem mass spectrometry. Psychoneuroendocrinology 38:50-57.

Muraven, M., Tice, D. M., \& Baumeister, R. F. (1998). Self-control as limited resource: Regulatory depletion patterns. Journal of Personality and Social Psychology, 74, $774-789$.

Muraven, M., and Baumeister, R.F. (2000). Self-regulation and depletion of limited resources: does self-control resemble a muscle? Psychological Bulletin, 126,247-259.

Otto, A. R., Raio, C. M., Chiang, A., Phelps, E. A., \& Daw, N. D. (2013). Workingmemory capacity protects model-based learning from stress. Proceedings of the National Academy of Sciences. 110(52), 20941-20946.

Petzold, A., Plessow, F., Goschke, T. \& Kirschbaum, C. (2010) Stress reduces use of negative feedback in a feedback-based learning task. Behavioral Neuroscience, 124, 248-255.

Schelling, T. C. (1984). Self-command in practice, in policy, and in a theory of rational choice. The American Economic Review, 74, 1-11. 
Schoofs D, Wolf OT, Smeets T (2009) Cold pressor stress impairs performance on working memory tasks requiring executive functions in healthy young men. Behav Neurosci 123(5):1066-1075.

Schwabe L, Wolf OT (2011) Stress-induced modulation of instrumental behavior: from goal-directed to habitual control of action. Behav Brain Research, 219(2):321-328.

Schwartz, J., Mochon, D., Wyper, L., Maroba, J., Patel, D., \& Ariely, D. (2014) Healthier by precommitment. Psychological Science, Vol. 25(2): 538-546.

Sinha R (2001) How does stress increase risk of drug abuse and relapse? Psychopharmacology (Berl), 158(4):343-359.

Shenhav, A. et al. (2013) The expected value of control: an integrative theory of anterior cingulate cortex function. Neuron, 79, 217-240

Soutschek, A., Ugazio, G., Crockett, M.J., Ruff, C.C., Kalenscher, T. and Tobler, P.N. (2017) Binding oneself to the mast: stimulating frontopolar cortex enhances precommitment. Social Cognitive and Affective Neuroscience, 12(4): 635-642.

Soutschek, A., \& Tobler, P. N. (2020). Know your weaknesses: Sophisticated impulsiveness motivates voluntary self-restrictions. Journal of Experimental Psychology: Learning, Memory, and Cognition, 46(9), 1611-1623.

Strotz (1956). Myopia and inconsistency in dynamic utility maximization. Review of Economic Studies, 23(3):165-80.

Thaler, R.H. \& Shefrin, H.M. (1981) An economic theory of self-control. Journal of Political Economics, 89:392-406.

Tversky, A. \& Thaler, R.H. Preference Reversals. Journal of Economic Perspectives, 4(2): 201-21.

Velasco M, Gómez J, Blanco M, Rodriguez I (1997) The cold pressor test: Pharmacological and therapeutic aspects. Am J Ther 4:34-38.

Westbrook. A. \& Brave, T.S. (2015) Cognitive effort: a neuroeconomic approach. Cognitive, Affective and Behavioral Neuroscience, 15, 395-415 